\title{
CRITERIA FOR THE EXISTENCE OF EQUIVARIANT FIBRATIONS ON ALGEBRAIC SURFACES AND HYPERKÄHLER MANIFOLDS AND EQUALITY OF AUTOMORPHISMS UP TO POWERS - A DYNAMICAL VIEWPOINT
}

\author{
FEI HU, JONGHAE KEUM, AND DE-QI ZHANG
}

\begin{abstract}
Let $X$ be a projective surface or a hyperkähler manifold and $G \leq \operatorname{Aut}(X)$. We give a necessary and sufficient condition for the existence of a non-trivial $G$-equivariant fibration on $X$. We also show that two automorphisms $g_{i}$ of positive entropy and polarized by the same nef divisor are the same up to powers, provided that either $X$ is not an abelian surface or the $g_{i}$ share at least one common periodic point. The surface case is known among experts, but we treat this case together with the hyperkähler case using the same language of hyperbolic lattice and following Ratcliffe [26] or Oguiso [23].

This arXiv version contains proofs omitted in the print version.
\end{abstract}

\section{INTRODUCTION}

We work over the field $\mathbb{C}$ of complex numbers. Let $X$ be a smooth projective variety or a compact Kähler manifold of dimension $n$. For a subgroup $G$ of the automorphism group $\operatorname{Aut}(X)$ of $X$ and a $G$-invariant subgroup $V$ of some cohomology group of $X$, we denote the induced action of $G$ on $V$ by $G \mid V$. For example the natural pullback action of $\operatorname{Aut}(X)$ on $H^{*}(X, \mathbb{C})$ is denoted by $\operatorname{Aut}(X) \mid H^{*}(X, \mathbb{C})$. For an automorphism $g \in \operatorname{Aut}(X)$, let

$$
\rho(g)=\rho\left(g^{*}\right):=\max \left\{|\lambda|: \lambda \text { is an eigenvalue of } g^{*} \mid \bigoplus_{i \geq 0} H^{i}(X, \mathbb{C})\right\}
$$

be the spectral radius of the pullback action $g^{*}$ on the total cohomology ring of $X$. We define the (topological) entropy as $h(g)=\log \rho(g)$. By the fundamental work of Gromov and Yomdim, the above definition is equivalent to the original dynamical definition of entropy (cf. [14], [27]). The $i$-th dynamical degree is defined as $d_{i}(g):=\rho\left(g^{*} \mid H^{i, i}(X, \mathbb{C})\right)$.

An element $g \in \operatorname{Aut}(X)$ is of null entropy (resp. positive entropy) if its entropy $h(g)=0$ (resp. $>0$ ). For a subgroup $G$ of $\operatorname{Aut}(X)$, we define the null-subset of $G$ as

$$
N(G):=\{g \in G: g \text { is of null entropy, i.e., } h(g)=0\} .
$$

In general, $N(G)$ may not be a subgroup of $G$. A group $G \leq \operatorname{Aut}(X)$ is of null entropy if every $g \in G$ is of null entropy, i.e., $G=N(G)$.

It is known that

$$
h(g)=\max _{0 \leq i \leq n} \log d_{i}(g)
$$

and $h(g)>0$ if and only if $d_{i}(g)>1$ for some (or equivalently for all) $i \in\{1, \ldots, n-1\}$; especially $h(g)>0$ if and only if $d_{1}(g)>1$. When $X$ is projective, $d_{1}(g)=\rho\left(g^{*} \mid \mathrm{NS}_{\mathbb{C}}(X)\right)$,

2010 Mathematics Subject Classification. 14J50, 32M05, 32H50, 37B40.

Key words and phrases. automorphism, complex dynamics, iteration, topological entropy. 
where $\mathrm{NS}(X)$ is the Néron-Severi group of $X$ and $\operatorname{NS}_{\mathbb{C}}(X):=\mathrm{NS}(X) \otimes_{\mathbb{Z}} \mathbb{C}$. In particular, for an automorphism $g$ of a projective surface $X$, we have

$$
\rho(g)=d_{1}(g)=\rho\left(g^{*} \mid \mathrm{NS}_{\mathbb{C}}(X)\right) ;
$$

see [9] and the references therein.

By the classification of surfaces, a compact complex surface $S$ has an automorphism of positive entropy only if $S$ is bimeromorphic to a rational surface, a $K 3$ surface, an Enriques surface or a complex torus (cf. [4]).

Let $X$ be a hyperkähler manifold. Thanks to Beauville, Bogomolov and Fujiki, there exists a natural bilinear (primitive) integral form $q_{X}$ of signature $\left(3, b_{2}-3\right)$ on the second integral cohomology group $H^{2}(X, \mathbb{Z})$. Moreover, if $X$ is projective, then the restriction of $q_{X}$ on the Néron-Severi group $\operatorname{NS}(X)$ is non-degenerate of signature $(1, \rho(X)-1)$, where $\rho(X)$ is the Picard number of $X$ (cf. [16]).

Hence algebraic surfaces and projective hyperkähler manifolds are very similar when we focus on their intersection forms: these forms are both represented by hyperbolic lattices.

Below is a hyperkähler analogue of Theorem 4.1 for surfaces. It uses the deep results due to Bayer - Macrì, Markman, Matsushita and Yoshioka (cf. [1], [20], [21] and [28]). Recall that a hyperkähler manifold is of type $K 3^{[n]}$ (resp. generalized Kummer) if it is deformation equivalent to $S^{[n]}$ for some $K 3$ surface $S$ (resp. to a generalized Kummer variety); see details from the above references. Since a bimeromorphic map $g$ on a hyperkähler manifold $X$ (or more generally a birational map on a minimal projective terminal variety) is isomorphic in codimension one (cf. [17]), it induces an isomorphism on $\mathrm{NS}(X)$. So we can define its first dynamical degree as $d_{1}(g):=\rho\left(g^{*} \mid \mathrm{NS}_{\mathbb{C}}(X)\right)$. We say $g$ (resp. $G$ ) is of null entropy if $d_{1}(g)=1$ (resp. $d_{1}(g)=1$ for every $g \in G$ ).

Theorem 1.1. Let $X$ be a $2 n$-dimensional projective hyperkähler manifold of type $K 3^{[n]}$ or of type generalized Kummer. Let $G$ be an infinite subgroup of $\operatorname{Bir}(X)$. Then $G$ is of null entropy if and only if there is a rational Lagrangian fibration (see 2.2) $\phi: X \rightarrow \mathbb{P}^{n}$ such that the birational action of $G$ on $X$ descends to a biregular action on $\mathbb{P}^{n}$, i.e., such that the following diagram commutes:

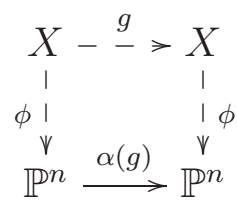

where $g$ is an element of $G$ and $\alpha$ is a group homomorphism from $G$ to $\operatorname{Aut}\left(\mathbb{P}^{n}\right)$.

A birational map $g \in \operatorname{Bir}(X)$ which is isomorphic in codimension one, is said to be polarized by a divisor $D$ if $g^{*} D \equiv \lambda D$ (numerical equivalence) for some $\lambda>0$.

Our next result is a hyperkähler analogue of Theorem 4.3 for surfaces. See Remark 4.4 for the converse of Theorem 1.2.

Theorem 1.2. Let $X$ be a projective hyperkähler manifold. Assume $D$ is a numerically nonzero $\mathbb{R}$-divisor such that $q_{X}(D) \geq 0$ for the Beauville-Bogomolov quadratic form $q_{X}$ 
(this holds when $D$ is movable or nef). Assume further that $g_{i} \in \operatorname{Bir}(X)(i=1,2)$ are of positive entropy and polarized by $D$. Then $g_{1}^{t_{1}}=g_{2}^{t_{2}}$ holds in $\operatorname{Bir}(X)$ for some $t_{i} \in \mathbb{Z} \backslash\{0\}$.

The first assertion below is due to [23, Theorem 2.1 (2) and Proposition 2.9]. Recall that a group $G$ is called virtually solvable (resp. virtually unipotent or virtually abelian), if a finite-index subgroup of $G$ is solvable (resp. unipotent or abelian). Note that (virtually) unipotent groups are defined only for linear algebraic groups. A group $G$ is called almost infinite cyclic, if a finite-index subgroup of $G$ is an infinite cyclic group.

Proposition 1.3. Let $X$ be a smooth projective surface and let $G \leq \operatorname{Aut}(X)$ be a group such that the induced action $G \mid \mathrm{NS}(X)$ is an infinite group, and $G$ is of null entropy. Then $G \mid \operatorname{NS}(X)$ is virtually abelian of rank $s \leq \rho(X)-2$. Further, $s \leq 18$, unless $X$ has Kodaira dimension $\kappa(X)=1$.

Remark 1.4. For a projective hyperkähler manifold $X$ the same proof in [23] implies the first assertion in Proposition 1.3. Further,

$$
s \leq \rho(X)-2 \leq \operatorname{dim} H^{1,1}(X, \mathbb{C})-2 \leq b_{2}(X)-4,
$$

with $b_{2}(X)$ the second Betti number of $X$. For a hyperkähler manifold $X$ of dimension four, it is known that $b_{2}(X) \in\{3, \ldots, 8,23\}$ (cf. [15, Main Theorem]).

We refer to [5], [6], [7], [10] and [25] for hard and deep results on birational actions on complex surfaces which are related to Theorem 4.1.

Acknowledgement. This paper is finalized when the last-named author visited KIAS, Seoul in December 2014. He thanks the institute for the support and warm hospitality. He is also supported by an ARF of NUS. The authors thank the referees for the valuable suggestions and references [6], [10], [15] and [26].

\section{Preliminaries}

Let $X$ be a smooth projective variety (resp. a compact Kähler manifold). The NéronSeveri group $\mathrm{NS}(X)$ is defined as the group of line bundles modulo algebraic equivalence. Denote its rank by $\rho(X)$, which is also called the Picard number of $X$. The Néron-Severi space $\mathrm{NS}_{\mathbb{R}}(X):=\mathrm{NS}(X) \otimes_{\mathbb{Z}} \mathbb{R}$ is the vector space of numerical equivalence classes of $\mathbb{R}$-divisors.

Denote the group of all automorphisms (resp. biholomorphisms) of $X$ by $\operatorname{Aut}(X)$. Denote the group of all birational maps (resp. bimeromorphisms) of $X$ by $\operatorname{Bir}(X)$. By $\operatorname{Aut}_{0}(X)$ we mean the identity connected component of $\operatorname{Aut}(X)$.

\subsection{Kähler cone, nef cone and movable cone}

Let $X$ be a compact Kähler manifold. Set $H^{1,1}(X, \mathbb{R}):=H^{1,1}(X, \mathbb{C}) \cap H^{2}(X, \mathbb{R})$. The Kähler cone $\mathcal{K}(X) \subseteq H^{1,1}(X, \mathbb{R})$ is the open convex cone of all Kähler classes on $X$. The closure of the Kähler cone $\mathcal{K}(X)$ in $H^{1,1}(X, \mathbb{R})$ is denoted by $\overline{\mathcal{K}}(X)$. The nef cone is defined as $\operatorname{Nef}(X):=\overline{\mathcal{K}}(X) \cap \mathrm{NS}_{\mathbb{R}}(X)$. An element $D$ in $\operatorname{Nef}(X)$ is called nef. 
A divisor $D$ in $\operatorname{NS}(X)$ is movable if the linear system $|D|$ has no fixed component, i.e., the base locus of this linear system has codimension at least two. The closure of all movable divisor classes in $\mathrm{NS}_{\mathbb{R}}(X)$ is called the movable cone of $X$ and denoted as $\overline{\operatorname{Mov}}(X)$. It is known that

$$
\operatorname{Nef}(X) \subseteq \overline{\operatorname{Mov}}(X) \subseteq \overline{\operatorname{Eff}}(X),
$$

where the pseudo-effective cone $\overline{\mathrm{Eff}}(X)$ of $X$ is the closure of all effective divisor classes in $\mathrm{NS}_{\mathbb{R}}(X)$.

\subsection{Rational Lagrangian fibrations on hyperkähler manifolds}

A hyperkähler manifold is a simply-connected compact Kähler manifold $X$ such that $H^{0}\left(X, \Omega_{X}^{2}\right)$ is generated by an everywhere non-degenerate holomorphic 2-form $\omega$. Note that $X$ is automatically of even complex dimensional (say $\operatorname{dim} X=2 n$ ). A surjective (holo)morphism $\phi: X \rightarrow S$ to a normal variety $S$ is said to be a Lagrangian fibration if a general fiber is connected and Lagrangian (i.e., the dimension of this fibre is $n$ and the restriction of the holomorphic 2-form $\omega$ to this fibre is trivial). A dominant meromorphic $\operatorname{map} \phi: X \rightarrow S$ to a normal variety $S$ is said to be a rational Lagrangian fibration if there exists a bimeromorphic map $\tau: X \rightarrow X^{\prime}$ to another hyperkähler manifold $X^{\prime}$ (which is necessarily isomorphic in codimension one) such that the composite map $\phi \circ \tau^{-1}: X^{\prime} \rightarrow S$ is a Lagrangian fibration. Thus $\phi^{*}$ is well-defined on the Néron-Severi group $\operatorname{NS}(S)$ of $S$.

\subsection{Positive cone and birational Kähler cone}

Let $X$ be a compact hyperkähler manifold. The positive cone $\mathcal{C}(X)$ in $H^{1,1}(X, \mathbb{R})$ is the connected component of the open cone

$$
\left\{\alpha \in H^{1,1}(X, \mathbb{R}): q_{X}(\alpha)>0\right\}
$$

that contains the Kähler cone $\mathcal{K}(X)$, where $q_{X}$ is the Beauville-Bogomolov quadratic form. The closure of $\mathcal{C}(X)$ in $H^{1,1}(X, \mathbb{R})$ is denoted by $\overline{\mathcal{C}}(X)$.

If $f: X^{\prime} \rightarrow X$ is a bimeromorphic map between two hyperkähler manifolds, then it is isomorphic in codimension one. Hence the pullback of $f$ is well defined on $H^{2}(X, \mathbb{C})$ and compatible with its Hodge structure, the Beauville-Bogomolov quadratic form $q_{X}$ and the birational Kähler cone $\mathcal{B K}(X)$ (cf. [17, Propositions 21.6 and 25.14]). Recall that the birational Kähler cone $\mathcal{B K}(X)$ of $X$ is defined as

$$
\mathcal{B K}(X):=\bigcup_{\tau: X-\rightarrow X^{\prime}} \tau^{*} \mathcal{K}\left(X^{\prime}\right),
$$

where $\tau: X \rightarrow X^{\prime}$ runs through all bimeromorphic maps from $X$ to another hyperkähler manifold $X^{\prime}$. Note that $\mathcal{B K}(X)$ is in general not a cone in $\mathcal{C}(X)$, but its closure $\overline{\mathcal{B K}}(X)$ in $H^{1,1}(X, \mathbb{R})$ is a closed convex cone contained in $\overline{\mathcal{C}}(X)$.

There is a geometric characterization of the birational Kähler cone, which states that $\alpha \in \overline{\mathcal{B K}}(X)$ if and only if $\alpha \in \overline{\mathcal{C}}(X)$ and $q_{X}(\alpha,[D]) \geq 0$ for all uniruled prime divisors $D \subset X$ (cf. [17, Proposition 28.7]). Furthermore, we have

$$
\overline{\operatorname{Mov}}(X)=\overline{\mathcal{B K}}(X) \cap \mathrm{NS}_{\mathbb{R}}(X) .
$$


The following two lemmas on group theory are useful in the future.

\section{Lemma 2.4.}

(1) Let $G$ be a group, $H \triangleleft G$ a finite normal subgroup and $g_{1}, g_{2} \in G$. Suppose that $\bar{g}_{1}=\bar{g}_{2}$ in $G / H$. Then there exists a positive integer s such that $g_{1}^{s}=g_{2}^{s}$.

(2) A group $G$ is almost infinite cyclic if and only if there is a finite-index subgroup $G_{1}$ of $G$ such that $G_{1} / G_{2}$ is infinite cyclic for some finite $G_{2} \triangleleft G_{1}$.

Proof. For part (1), by the assumption, $g_{1}^{n} g_{2}^{-n} \in H$ for all $n \in \mathbb{Z}$. Since $H$ is finite, there exist $m<n$ such that $g_{1}^{m} g_{2}^{-m}=g_{1}^{n} g_{2}^{-n}$. Then $g_{1}^{s}=g_{2}^{s}$ for $s:=n-m$.

Part (2) is easy; see [3, Lemma 2.4].

Note that the lemma below or [3, Lemma 2.7] has been generalized in [8, Lemma 5.5] without assuming $G$ to be a subgroup of an algebraic group. But the following simple form with simple proof as in [3, Lemma 2.7] is enough for us.

Lemma 2.5. Let $G$ be a subgroup of an algebraic group $\hat{G}$ (which is an extension of an abelian variety by a linear algebraic group). Consider the exact sequence

$$
1 \rightarrow N \rightarrow G \rightarrow Q \rightarrow 1
$$

Suppose that both $N$ and $Q$ are virtually solvable. Then so is $G$.

Proof. This is implicitly proved in [3, Lemma 2.7]. We just use the fact that the Zariskiclosure of $N$ in $\hat{G}$ has only finitely many connected components. Then the same argument in [3] gives the proof.

At the end of this section we quote the following lemmas which will be used later.

Lemma 2.6 (cf. [12, Theorem 4.8], [19, Proposition 2.2], or [3, Lemma 2.6]). Let $X$ be a smooth projective variety (resp. a compact Kähler manifold), and $L:=\mathrm{NS}(X) /($ torsion) $\left(\right.$ resp. $H^{2}(X, \mathbb{Z}) /($ torsion$\left.)\right)$. Then a group $G \leq \operatorname{Aut}(X)$ has finite induced action $G \mid L$ if and only if the index $\left|G: G \cap \operatorname{Aut}_{0}(X)\right|$ is finite.

Lemma 2.7 (cf. [24, Corollary 2.7], or [16, §9] for the case of the action of $G$ on $H^{2}(X, \mathbb{Z})$ ). Let $X$ be a projective hyperkähler manifold and let $G \leq \operatorname{Bir}(X)$. Then the homomorphism $r_{\mathrm{NS}}: G \rightarrow \mathrm{O}(L)$ (cf. 3.1), $g \mapsto g^{*} \mid L$ has finite kernel, where $L:=\mathrm{NS}(X)$.

Lemma 2.8 (cf. [11, Application 1.4]). Let $X$ be a compact Kähler surface with first Betti number $b_{1}(X)=0$. Suppose that $\operatorname{Aut}_{0}(X) \neq 1$. Then $\left|\operatorname{Aut}(X): \operatorname{Aut}_{0}(X)\right|<\infty$ and $X$ is projective. In particular, $\operatorname{Aut}(X)$ is of null entropy.

\section{Automorphisms of hyperbolic lattices And APplications}

\subsection{Hyperbolic lattices and their symmetries}

By a lattice $L$, we mean a free abelian group $L \cong \mathbb{Z}^{\oplus r}$ admitting a non-degenerate symmetric bilinear form $L \times L \rightarrow \mathbb{Z}$, denoted as $(\cdot, \cdot)$. The signature of $L$ is defined as 
the signature of $L_{\mathbb{R}}:=L \otimes_{\mathbb{Z}} \mathbb{R}$. The lattice $L$ is called hyperbolic if the signature of $L$ is $(1, r-1)$.

The positive cone $\mathcal{C}\left(L_{\mathbb{R}}\right)$ is one of the two connected components of

$$
\left\{x \in L_{\mathbb{R}}:(x, x)>0\right\} .
$$

Denote the boundary (resp. closure) of $\mathcal{C}\left(L_{\mathbb{R}}\right)$ by $\partial \mathcal{C}\left(L_{\mathbb{R}}\right)$ (resp. $\overline{\mathcal{C}}\left(L_{\mathbb{R}}\right)$ ). Note that there is no ambiguity when applied to surfaces or hyperkähler manifolds. In fact, the positive cone is always chosen by us so that it contains an ample class or a Kähler class.

We denote the group of isometries of $L$ by

$$
\mathrm{O}(L):=\{g \in \operatorname{Aut}(L):(g x, g y)=(x, y), \forall x, y \in L\} .
$$

The subgroup $\mathrm{O}(L)^{\prime}$ of $\mathrm{O}(L)$ which preserves the positive cone has index two.

Remark 3.2. When $X$ is a smooth projective surface (resp. a projective hyperkähler manifold), the intersection form (resp. the Beauville-Bogomolov quadratic form $q_{X}$ ) on $X$ gives a non-degenerate symmetric bilinear form on the lattice $L:=\mathrm{NS}(X) /$ (torsion) (resp. $L:=\mathrm{NS}(X)$; note that the Néron-Severi group is torsion free since $X$ is simply connected). In either case, $L$ is a hyperbolic lattice of signature $(1, \rho(X)-1)$ (cf. [17, Proposition 26.13]). The above $L$ is called the Néron-Severi lattice of $X$.

\subsection{Spectral radius and entropy}

Let $k=\mathbb{Z}$ or a subfield of $\mathbb{C}, V$ a finite $k$-module, and $\varphi: V \rightarrow V$ a $k$-linear endomorphism. Set $V_{\mathbb{C}}:=V \otimes_{k} \mathbb{C}$. Use the same $\varphi$ to denote its extension to a $\mathbb{C}$-linear endomorphism $\varphi: V_{\mathbb{C}} \rightarrow V_{\mathbb{C}}$. Define the spectral radius of $\varphi$ as follows

$$
\rho(\varphi):=\max \left\{|\lambda|: \lambda \in \mathbb{C} \text { is an eigenvalue of } \varphi: V_{\mathbb{C}} \rightarrow V_{\mathbb{C}}\right\}
$$

Define the entropy of $\varphi$ as $h(\varphi):=\log \rho(\varphi)$.

Let $G \leq \mathrm{O}(L)$ for some lattice $L$. We define the null-subset of $G$ as

$$
N(G):=\{g \in G: g \text { is of null entropy, i.e., } h(g)=0 \text {, or equivalently } \rho(g)=1\} .
$$

The following generalized Perron-Frobenius theorem is due to Birkhoff.

Lemma 3.4 (cf. [2]). Let $C$ be a strictly (i.e., salient) convex closed cone of a finitedimensional $\mathbb{R}$-vector space $V$ such that $C$ spans $V$ as a vector space. Let $g: V \rightarrow V$ be a $\mathbb{R}$-linear endomorphism such that $g(C) \subseteq C$. Then the spectral radius $\rho(g)$ is an eigenvalue of $g$ and there is an eigenvector $v_{g} \in C$ corresponding to the eigenvalue $\rho(g)$.

We now prepare some general results on $\mathrm{O}(L)$ for a hyperbolic lattice $L$.

Lemma 3.5 (cf. [26, Chapter 5], or [23, Proposition 2.2], or [3, Theorem 2.2]). Let L be a hyperbolic lattice. Suppose that $G \leq \mathrm{O}(L)^{\prime}$ is of null entropy. Then $G$ contains $U$ as a finite-index normal subgroup, where

$$
U=U(G):=\left\{g \in G: g \text { is unipotent as an element in } \operatorname{GL}\left(L_{\mathbb{C}}\right)\right\} .
$$


Proof. This is implicitly proved in [23, Proposition 2.2]. Indeed, by [23, Lemma 2.5], $U$ is a subgroup of (and hence normal in) $G$. Replacing $G$ by a finite-index subgroup, we may assume that the Zariski-closure of $G$ in $\operatorname{GL}\left(L_{\mathbb{C}}\right)$ is connected. Now the quotient group $G / U$ can be embedded into an algebraic torus (and hence in some general linear group) and every element of it is of finite order (bounded by a constant depending only on $\operatorname{rank}(L))$ by Kronecker's theorem. Note also that every subgroup of a general linear group over $\mathbb{C}$ with bounded exponent is a finite group by the classical Burnside's Theorem. Hence $|G: U|<\infty$ as required.

The result below follows from the classification of elliptic, parabolic or loxodromic elements; see for instance [6], or the proof of [29, Lemma 2.12].

Lemma 3.6 (cf. [6], or proof of [29, Lemma 2.12]). Let $L$ be a hyperbolic lattice with $\mathcal{C}\left(L_{\mathbb{R}}\right)$ the positive cone. Let $g \in \mathrm{O}(L)^{\prime}$ such that $g(v)=\lambda v$ for some $0 \neq v \in \overline{\mathcal{C}}\left(L_{\mathbb{R}}\right)$.

(1) If $\lambda=1$, then $g$ is of null entropy.

(2) If $\lambda>1$ (resp. $<1$ ), then $g$ is of positive entropy, $v$ is parallel to the $v_{g}$ (resp. $\left.v_{g^{-1}}\right)$ in Lemma 3.4 and $\rho(g)=\lambda$ (resp. $\left.\rho\left(g^{-1}\right)=\lambda^{-1}\right)$.

(3) Suppose that $\lambda \neq 1$. Then $\rho(g)$ is a Salem number or a quadratic integer (and hence an irrational algebraic integer), the $v_{g}$ in Lemma 3.4 in the current case is unique up to a scalar, and $\rho(g)=\rho\left(g^{-1}\right)$.

Proof. For the assertions (1) and (2), the proof is similar to [29, Lemma 2.12]. We only have to consider the case where $g$ is of positive entropy. Hence $g^{-1}$ is also of positive entropy, because $\operatorname{det}(g)= \pm 1$ and $g$ is defined over the integral lattice $L$. By Lemma 3.4, there are nonzero eigenvectors $v_{g^{ \pm 1}} \in \overline{\mathcal{C}}\left(L_{\mathbb{R}}\right)$ such that

$$
g^{ \pm 1}\left(v_{g^{ \pm 1}}\right)=\rho\left(g^{ \pm 1}\right) v_{g^{ \pm 1}}
$$

It suffices to show the claim that $v$ is parallel to one of $v_{g^{ \pm 1}}$. Suppose the contrary that this claim is not true. The hyperbolicity of $L$ and the Hodge index type theorem imply that

$$
0<\left(v, v_{g}\right)=\left(g(v), g\left(v_{g}\right)\right)=\lambda \rho(g)\left(v, v_{g}\right) .
$$

Hence $\lambda=1 / \rho(g)<1$. By the same reasoning,

$$
0<\left(v, v_{g^{-1}}\right)=\left(g^{-1}(v), g^{-1}\left(v_{g^{-1}}\right)\right)=\lambda^{-1} \rho\left(g^{-1}\right)\left(v, v_{g^{-1}}\right) .
$$

Hence $\lambda=\rho\left(g^{-1}\right)>1$, contradicting the previous outcome. Thus the claim is true. Hence the assertions (1) and (2) are true.

For the assertion $(3), \rho(g)$ is a Salem number by [22, Proposition 2.5]. Hence $\rho(g)=$ $\rho\left(g^{-1}\right)$. The uniqueness follows from the above argument for assertion (2).

Lemma 3.7 (cf. [26, §5.5] or [23, Theorem 2.1]). Let $L$ be a hyperbolic lattice and let $G \leq \mathrm{O}(L)^{\prime}$. Suppose that $G$ is of null entropy and $G$ is an infinite group. Then we have:

(1) There is a unique (up to scalars) nonzero $v \in \overline{\mathcal{C}}\left(L_{\mathbb{R}}\right)$ such that $g(v)=v$ for all $g \in G$. Moreover, $v^{2}=0$ and $v$ can be taken to be in the integral lattice $L$. 
(2) Suppose that $W$ is a non-trivial G-invariant closed subcone of $\overline{\mathcal{C}}\left(L_{\mathbb{R}}\right)$. Then $v$ belongs to $W$.

(3) If $L=\mathrm{NS}(X) /$ (torsion) (resp. $\mathrm{NS}(X)$ ) for some smooth projective surface (resp. projective hyperkähler manifold) $X$ and the above $G$ equals $H \mid L$ for some group $H \leq \operatorname{Aut}(X)$, then $v$ is a nef divisor class.

(4) If $L=\mathrm{NS}(X)$ for some projective hyperkähler manifold $X$ and the above $G$ equals $H \mid L$ for some group $H \leq \operatorname{Bir}(X)$, then $v$ belongs to the movable cone $\overline{\operatorname{Mov}}(X)$.

Proof. Part (1) is contained in [26, §5.5] or [23]. Indeed, [23, Lemma 2.8] implies the claim that there is no $v \in L_{\mathbb{Q}}$ such that $v^{2}>0$ and $g(v)=v$ for all $g \in G$. There is also no such $v$ in $L_{\mathbb{R}}$. Indeed, as noticed in [23], the eigenspace $V(g, 1)$ of eigenvalue 1 is defined over $\mathbb{Q}$, hence the intersection $\cap_{g \in G} V(g, 1)$ is also defined over $\mathbb{Q}$. Thus the existence of such $v$ in $L_{\mathbb{R}}$ (and the density of $\mathbb{Q}$ in $\mathbb{R}$ ) would imply the same for a $v$ in $L_{\mathbb{Q}}$, contradicting the claim. By the claim above and [23, Lemma 2.8] there indeed exists a unique nonzero ray $\mathbb{R}_{>0} v \subseteq \overline{\mathcal{C}}\left(L_{\mathbb{R}}\right)$ such that $g(v)=v$ for all $g \in G$; further $v^{2}=0$ and $v$ can be taken to be in $L$.

Parts (3) and (4) are consequences of part (2) applied to the nef cone $\operatorname{Nef}(X)$ and the movable cone $\overline{\operatorname{Mov}}(X)$, respectively. We also note that a bimeromorphic map of a hyperkähler manifold $X$ is an isomorphism in codimension one, hence induces an isomorphism of $\mathrm{NS}(X)$ and preserves the movable cone $\overline{\operatorname{Mov}}(X)$.

For part (2), note that $G$ is virtually unipotent and hence virtually solvable (cf. [23, Proof of Theorem 2.1] or [3, Proof of Theorem 2.2]). Thus $G_{1}\left(v^{\prime}\right) \subseteq \mathbb{R}_{>0} v^{\prime}$ for some nonzero element $v^{\prime} \in W$ and a finite-index subgroup $G_{1}$ of $G$, by the cone theorem of Lie-Kolchin type (cf. [18, Theorem 1.1]). By Lemma 3.6 and the assumption $G=N(G)$, we have $G_{1}\left(v^{\prime}\right)=v^{\prime}$. Now apply the uniqueness property in part (1) to $G_{1}$, we conclude that $v \in \mathbb{R}_{>0} v^{\prime} \subseteq W$. This proves part (2) and the whole lemma.

Lemma 3.8. Let $L$ be a hyperbolic lattice and let $G \leq \mathrm{O}(L)^{\prime}$. Suppose that the null-subset $N(G)$ is a subgroup of (and hence normal in) $G$ and $G \neq N(G)$. Then $N(G)$ is finite.

Proof. Suppose the contrary that $|N(G)|=\infty$. By Lemma 3.7 or [23, Lemma 2.8], there is a nonzero $v \in \partial \mathcal{C}\left(L_{\mathbb{R}}\right)$ such that $h(v)=v$ for any $h \in N(G)$. Further, the ray $\mathbb{R}_{>0} v \subseteq \partial \mathcal{C}\left(L_{\mathbb{R}}\right)$ is unique and defined over $\mathbb{Z}$. Now for any $g \in G$ and $h \in N(G)$, we must have $h(g(v))=g(v)$ since $N(G) \triangleleft G$. Thus $g(v)=r_{g} v$ for some $r_{g} \in \mathbb{R}_{>0}$ by the uniqueness of the above ray. Moreover, $r_{g}$ must be rational since $v$ is in the integral lattice $L$. Take one $g \in G$ which is of positive entropy. Since $g(v)=r_{g} v$, we have $r_{g}=\rho(g)^{ \pm 1}$ by Lemma 3.6. But $\rho(g)$ is a Salem number or a quadratic integer and hence is irrational, contradicting the rationality of $r_{g}$. Hence $N(G)$ is finite.

The result below is contained in [26, Theorems 5.5.9- 5.5.10]. See also [6, Theorem 3.2] or [29, Theorem 3.1] for $\mathrm{NS}(X)$ of a surface $X$.

Lemma 3.9 (cf. [26, Theorems 5.5.9 - 5.5.10]). Let $L$ be a hyperbolic lattice and let $G \leq \mathrm{O}(L)^{\prime}$. Then the following are equivalent:

(1) $G$ is virtually solvable. 
(2) Replacing $G$ by a finite-index subgroup, there is a real vector $v \in \overline{\mathcal{C}}\left(L_{\mathbb{R}}\right) \backslash\{0\}$ such that $G(v) \subseteq \mathbb{R}_{>0} v$.

(3) Replacing $G$ by a finite-index subgroup, we have $N(G) \triangleleft G$ and $G / N(G) \cong \mathbb{Z}^{\oplus r}$ for some nonnegative integer $r \leq 1$.

Proof. For readers' convenience, we include the proof.

$(1) \Rightarrow(2)$ Replacing $G$ by a finite-index subgroup, we may assume that the Zariski closure of $G \mid L_{\mathbb{C}}$ in $\operatorname{GL}\left(L_{\mathbb{C}}\right)$ is connected. Then the assertion (2) is just a consequence of Theorem of Lie-Kolchin type for a cone (cf. [18, Corollary 2.3]).

$(2) \Rightarrow(3)$ Replacing $G$, we may assume that there exists a $\lambda_{g} \in \mathbb{R}_{>0}$ such that $g(v)=$ $\lambda_{g} v$ for any $g \in G$. Define a group homomorphism

$$
\phi: G \rightarrow(\mathbb{R},+), g \mapsto \log \lambda_{g} .
$$

Now the assertion (3) follows from the two claims below.

Claim 3.10. Ker $\phi=N(G)=\{g \in G: \rho(g)=1\}$. In particular, $N(G) \triangleleft G$.

Proof. Clearly, $N(G) \subseteq \operatorname{ker} \phi$. Conversely, suppose $g \in \operatorname{ker} \phi$. Then $g(v)=v$. Hence $g$ is of null entropy, i.e., $g \in N(G)$, by Lemma 3.6.

Claim 3.11. $\phi(G)$ is discrete in the additive group $\mathbb{R}$, hence $\phi(G)=0$ or $\mathbb{Z}$.

Proof. It suffices to show that 0 is an isolated point in $\phi(G)$. For any fixed positive real number $\delta$, consider the set

$$
G_{\delta}:=\left\{g \in G:\left|\log \lambda_{g}\right|<\delta\right\} .
$$

For any $g \in G_{\delta}, \lambda_{g}^{ \pm 1}$ is bounded by $e^{\delta}$. If $g \in N(G)$, then $\lambda_{g}=1$. Suppose that $g$ is of positive entropy. Then $\lambda_{g} \neq 1$ and $\rho(g)=\lambda_{g}^{ \pm 1}$ (cf. Lemma 3.6). The minimal polynomial of $g$ is a Salem polynomial with two real roots $\rho(g)^{ \pm 1}$ and other roots on the unit circle (cf. [22, Proposition 2.5], [29, Lemma 2.7]). Hence the coefficients of these minimal polynomials are all bounded. Note that these minimal polynomials are defined over $\mathbb{Z}$ with degree bounded by $\operatorname{rank}(L)$. So there are only finitely many such minimal polynomials for all $g \in G_{\delta}$. Therefore, the set of all eigenvalues (especially $\lambda_{g}$ ) of such $g \in G_{\delta}$ is finite. Thus 0 is an isolated point in $\phi(G)$.

$(3) \Rightarrow(1)$ Replacing $G$, we may assume that $G / N(G)$ is cyclic and hence solvable. $N(G)$ is virtually unipotent by Lemma 3.5 and hence virtually solvable. Thus $G$, regarded as a subgroup of $\operatorname{GL}\left(L_{\mathbb{C}}\right)$ is virtually solvable, by Lemma 2.5. So the assertion (1) is true.

Corollary 3.12. Assume one of the equivalent conditions in Lemma 3.9 holds and further that $G \neq N(G)$. Then replacing $G$ by a finite-index subgroup, we have $N(G)$ is finite and $G$ is almost infinite cyclic.

Proof. By the assumption and Lemma 3.9, we may assume that $G / N(G) \cong \mathbb{Z}$ after replacing $G$. Since $G \neq N(G)$, our $N(G)$ is finite by Lemma 3.8. Hence $G$ is almost infinite cyclic; see Lemma 2.4 . 
The following result is a direct consequence of [26]. And it is the lattice-theoretical counterpart of the result on surfaces or hyperkähler manifolds we will state later on.

Proposition 3.13 (cf. [26, Theorem 5.5.8]). Let $L$ be a hyperbolic lattice and let $g_{1}, g_{2} \in$ $\mathrm{O}(L)^{\prime}$. Suppose that both $g_{i}$ are of positive entropy, and there is a nonzero element $v \in \overline{\mathcal{C}}\left(L_{\mathbb{R}}\right)$, such that $g_{i}(v)=\lambda_{i} v$ for some real numbers $\lambda_{i}>0$. Then $g_{1}^{t_{1}}=g_{2}^{t_{2}}$ for some $t_{i} \in \mathbb{Z} \backslash\{0\}$.

Proof. This follows from [26, Theorem 5.5.8]. We prove it for the convenience of readers. Let $G=\left\langle g_{1}, g_{2}\right\rangle$. Then $G$ has a common real eigenvector $v \in \overline{\mathcal{C}}\left(L_{\mathbb{R}}\right) \backslash\{0\}$ and $G \neq N(G)$. Applying Lemma 3.9 and replacing $G$ by a finite-index subgroup, we have

$$
\pi: G \rightarrow G / N(G) \cong \mathbb{Z}
$$

Further, $N(G)$ is finite by Lemma 3.8. Thus $\pi\left(g_{1}^{n_{1}}\right)=\pi\left(g_{2}^{n_{2}}\right)$ for some $n_{1}, n_{2} \in \mathbb{Z} \backslash\{0\}$. Now the proposition follows from Lemma 2.4 .

\subsection{Consequences on automorphisms of surfaces and hyperkähler manifolds}

Now we apply the above results on hyperbolic lattice to the Néron-Severi lattice (see Remark 3.2) of a projective surface or a hyperkähler manifold.

Proposition 3.15. Let $X$ be a smooth projective surface and $G \leq \operatorname{Aut}(X)$. Suppose the null-subset $N(G)$ is a proper subgroup of (and hence normal in) $G$. Then we have:

(1) $N(G) \mid \mathrm{NS}(X)$ is a finite group.

(2) Suppose that $|N(G)|=\infty$. Then $X$ is an abelian surface, $H:=N(G) \cap \operatorname{Aut}_{0}(X)=$ $G \cap \operatorname{Aut}_{0}(X)$ is Zariski-dense in $\operatorname{Aut}_{0}(X)(\cong X)$ and the index $|N(G): H|<\infty$.

Proof. Set $L:=\mathrm{NS}(X) /($ torsion). Replacing $G$ by a suitable finite-index subgroup we may assume that the Zariski-closure of $G \mid L_{\mathbb{C}}$ in $\operatorname{GL}\left(L_{\mathbb{C}}\right)$ is connected. Note that $N(G)$ is the inverse of $N(G \mid L)$ via the natural homomorphism $G \rightarrow G \mid L \leq \mathrm{O}(L)^{\prime}$, and $N(G) \mid L=$ $N(G \mid L)$. Part (1) follows directly from Lemma 3.8.

For part (2), suppose that $N(G)$ is infinite (but $N(G) \mid L$ is finite by part (1)). Then the Kodaira dimension $\kappa(X) \leq 1$ since the automorphism group of a variety of general type is finite. Also $H:=N(G) \cap \operatorname{Aut}_{0}(X)$ is equal to $G \cap \operatorname{Aut}_{0}(X)$ and has finite-index in $N(G)$, by Lemma 2.6. Thus $\operatorname{Aut}_{0}(X) \neq 1$, so $X$ is neither birational to a $K 3$ surface nor to an Enriques surface. Since $G \neq N(G)$, our $X$ is not a rational surface by Lemma 2.8 .

The assumption $G \neq N(G)$ and Lemma 3.6 or [29, Lemma 2.12] imply that no fibration on $X$ is equivariant under the action of $G$ or its finite-index subgroup, and hence $X$ is birational to an abelian surface. (You may also appeal to Cantat's classification of surfaces with at least one automorphism of positive entropy; see [4].)

Let $G_{0}$ be the Zariski closure of $H$ in $\operatorname{Aut}_{0}(X)$. Then $G_{0}$ is normalized by $G$. Thus $X$ is dominated by $G_{0}$, by the proof of [31, Lemma 2.14] and since $X$ and hence its $G$ equivariant blowup have no non-trivial $G$-equivariant fibration. Note that the albanese map $\operatorname{alb}_{X}: X \rightarrow A:=\operatorname{Alb}(X)$ is surjective, birational and necessarily $\operatorname{Aut}(X)$-equivalent. 
$G_{0}$ induces an action on $A$ and we denote it by $G_{0} \mid A$. Since $G_{0} \mid A$ also has a Zariski-dense open orbit in $A$, the closedness of $G_{0}$ implies that $G_{0} \mid A=\operatorname{Aut}_{0}(X)(\cong A)$. Let $B \subset A$ be the locus over which alb $\mathrm{al}_{X}$ is not an isomorphism. Note that $B$ and $\operatorname{alb}_{X}^{-1}(B)$ are $G_{0}$-stable. Since $G_{0} \mid A=\operatorname{Aut}_{0}(X)$ consists of all translations on $A$, we have $B=\emptyset$. Thus $X$ is an abelian surface. This proves Proposition 3.15.

Below are consequences of Proposition 3.15 and hyperkähler analogue. Theorem 3.16 is part of [6, Theorem 3.2 and Remark 3.3]. It also follows from Lemmas 3.8 and 3.9 and Lemma 2.4. The proof of Theorem 3.17 is similar to the part (1) of Theorem 3.16, but Lemma 2.7 is also used.

Theorem 3.16 (cf. [6, Theorem 3.2, Remark 3.3]). Let $X$ be a smooth projective surface. Suppose that $G \leq \operatorname{Aut}(X)$ is not of null entropy and that $G \mid \mathrm{NS}_{\mathbb{C}}(X)$ is virtually solvable. Then after replacing $G$ by a finite-index subgroup, we have:

(1) $N(G) \mid \mathrm{NS}(X)$ is finite and $G \mid \mathrm{NS}(X)$ is almost infinite cyclic.

(2) $G$ is almost infinite cyclic, unless $X$ is an abelian surface and $G \cap \operatorname{Aut}_{0}(X)$ is Zariski-dense in $\operatorname{Aut}_{0}(X)$.

Proof. For readers' convenience, we include the proof. Let $L=\mathrm{NS}(X) /$ (torsion). Note that $N(G)$ is the inverse of $N(G \mid L)$ via the natural homomorphism $G \rightarrow G \mid L$, and $N(G) \mid L=N(G \mid L)$. Replacing $G$ by a finite-index subgroup, we may assume that $G \mid L$ is solvable and the Zariski closure of $G \mid L_{\mathbb{C}}$ in $\operatorname{GL}\left(L_{\mathbb{C}}\right)$ is connected. Then, as in Lemma 3.9, $N(G \mid L) \triangleleft G \mid L$ and

$$
G / N(G) \cong(G \mid L) /(N(G \mid L)) \cong \mathbb{Z}
$$

Since $N(G) \neq G$, Lemma 3.8 implies that $N(G) \mid L$ and hence $N(G) \mid \mathrm{NS}(X)$ are finite. Hence part (1) is true; see Lemma 2.4.

If $N(G)$ is finite, then $G$ is almost infinite cyclic by Lemma 2.4. Otherwise, we can apply Proposition 3.15 and conclude part (2).

Theorem 3.17. Let $X$ be a projective hyperkähler manifold. Suppose that $G \leq \operatorname{Bir}(X)$ is not of null entropy and that $G \mid \mathrm{NS}_{\mathbb{C}}(X)$ is virtually solvable. Then after replacing $G$ by a finite-index subgroup, $N(G)$ is finite and $G$ is almost infinite cyclic.

Proof. We argue as in Theorem 3.16 (1) and also apply Lemma 2.7.

\section{Results For SuRfaCES AND PROOFs of RESUlts in the INTRODUCTION}

For a surface $X$, a group $G \leq \operatorname{Aut}(X)$ being of null entropy has a clear geometric interpretation as follows. The crucial case has been dealt with in [13, Theorem 2] and [23, Theorem 2.1]. [6, Theorem 2.11] deals with the cyclic group case, but the general case is similar due to the canonicity of the fibrations involved. We state it for comparison with Theorem 1.1.

Theorem 4.1 (cf. [6, Theorem 2.11]). Let $X$ be a smooth projective surface and let $G \leq \operatorname{Aut}(X)$ be a group such that the induced action $G \mid \mathrm{NS}(X)$ is an infinite group. 
Then $G$ is of null entropy if and only if there is a $G$-equivariant fibration $X \rightarrow B$ onto a nonsingular projective curve $B$.

The remark below shows that one can also handle the case where $G$ is infinite while $G \mid \mathrm{NS}(X)$ is finite. See neat and exhaustive results in [25] when $G \leq \operatorname{Bir}(X)$ is cyclic.

Remark 4.2. Suppose that $X$ is a compact Kähler manifold (resp. a smooth projective variety) and $G \leq \operatorname{Aut}(X)$ is infinite while $G \mid H^{1,1}(X, \mathbb{C})$ (resp. $G \mid \mathrm{NS}(X)$ ) is finite. By Lemma 2.6 and its proof in [3, Lemma 2.6], $G_{0}:=G \cap \operatorname{Aut}_{0}(X)$ is infinite and has finite index in $G$. Let $\bar{G}_{0}$ be the Zariski-closure of $G_{0}$ in $\operatorname{Aut}_{0}(X)$, and $L\left(\bar{G}_{0}\right)$ the linear part of $\bar{G}_{0}$; see [12] or [19]. Then either the graph of the quotient map $X \rightarrow X / \bar{G}_{0}$ or $X \rightarrow-$ $X / L\left(\bar{G}_{0}\right)$ as in [12, Lemma 4.2] gives a non-trivial $G$-equivariant fibration, or $X$ is almost homogeneous under the action of $L\left(\bar{G}_{0}\right)$, or the albanese map $\operatorname{alb}_{X}: X \rightarrow A:=\operatorname{Alb}(X)$ is $\operatorname{Aut}(X)$ - (and hence $\bar{G}_{0^{-}}$) equivariant bimeromophic with $\bar{G}_{0}$ acting densely on both the domain and codomain. In the second case, $X$ is a unirational variety with $-K_{X}$ big and $\left|\operatorname{Aut}(X): \operatorname{Aut}_{0}(X)\right|<\infty$ (cf. [11, Theorem 1.2]). In the last case, the exceptional locus of $X \rightarrow A$ (resp. its image in $\operatorname{Alb}(X))$ is stable under the action of $\bar{G}_{0} \leq \operatorname{Aut}_{0}(X)$ (resp. $\bar{G}_{0}=\operatorname{Aut}_{0}(A) \cong A$ ), hence this locus is empty, i.e., $X \rightarrow A$ is an isomorphism.

Proof of Theorem 4.1 (for the convenience of readers). Set $L:=\mathrm{NS}(X) /$ (torsion).

For the 'if' part: Take a fibre $F$ of the fibration $X \rightarrow B$. The $G$-equivariance of the fibration implies that $g^{*}[F]=[F]$ for every $g \in G$. Hence $G$ is of null entropy by [29, Lemma 2.12] or Lemma 3.6.

For the 'only if' part: Suppose that $|G| L \mid=\infty$ and $G$ is of null entropy. Thus $X$ is not of general type and hence the Kodaira dimension $\kappa(X) \leq 1$. By Lemma 3.7 or [23, Theorem 2.1], there is a unique (up to scalars) nonzero nef divisor $D$ with $D^{2}=0$ and $g^{*}[D]=[D]$ for all $g \in G$. Further $D$ can be chosen to be integral.

If $\kappa(X)=1$ (i.e., properly elliptic surfaces), or $X$ is a hyperelliptic surface and hence has a unique elliptic fibration onto $\operatorname{Alb}(X)$, or $X$ is an irrational ruled surface, then $X$ has a typical fibration which is clearly $G$-equivariant.

It remains to consider (blowups of) $K 3$, Enriques, abelian and rational surfaces. We may assume that $X$ is minimal unless it is rational.

If $X$ is a $K 3$ surface then the Riemann-Roch theorem implies that the above nef $D$ is parallel to a fibre of an elliptic fibration and Theorem 4.1 is true. The Enriques case can be reduced to its $K 3$ cover and the pullback of $D$.

Suppose that $X$ is rational. We may assume that the pair $(X, G)$ is minimal. Then, by [13, Theorem 2 , and p. 104], $K_{X}^{2}=0$, the Iitaka $D$-dimension $\kappa\left(X,-K_{X}\right)=1$ and for some $m \geq 1,\left|-m K_{X}\right|$ defines an elliptic fibration which is clearly $G$-equivariant.

Finally, suppose that $X$ is an abelian surface. By Lemma 3.5, $G \mid L$ is virtually unipotent. Since $G \mid L$ is infinite, some $g_{0} \in G$ restricts to a unipotent element $g_{0}^{*} \mid L$ which is non-trivial and hence of infinite order. Since $G$ is of null entropy, we may also assume that $g_{0}^{*} \mid H^{0}\left(X, \Omega_{X}^{1}\right)$ is non-trivial and unipotent, after replacing $g_{0}$ by its power and using Kronecker's theorem. Write $g_{0}=T_{a} \circ h$ with $T_{a}$ a translation and $h$ a group automorphism. Then $\operatorname{ker}\left(h-\mathrm{id}_{X}\right)$ contains a 1-dimensional subtorus $F$, an elliptic curve. Hence 
$g_{0}$ permutes cosets of $X / F$, and $g_{0}^{*}[F]=[F]$; see the calculation in [30, Lemma 2.15]. Since $g_{0}^{*}[D]=[D]$, the condition $\left|\left\langle g_{0}\right\rangle\right| L \mid=\infty$ and the uniqueness in Lemma 3.7 imply that $[D]=[F]$ after replacing $D$ by a multiple. Thus, $g^{*}[F]=[F]$ for all $g \in G$. So $g(F)$ is also a fibre of $X \rightarrow X / F$, otherwise, $0<(g(F), F)=(F, F)=0$, a contradiction. Hence $X \rightarrow X / F$ is a $G$-equivariant fibration. Theorem 4.1 is proved.

The base variety of a Lagrangian fibration is known to be a projective space when it is smooth. This is the case in the situation of Theorem 1.1.

Proof of Theorem 1.1. Set $L:=\mathrm{NS}(X)$ as before.

For the 'if' part: Take a hyperplane $H$ on $\mathbb{P}^{n}$, and denote its pullback $\phi^{*} H$ by $P$. Then we have $g^{*} P=\phi^{*} \alpha(g)^{*} H$. Since $\alpha(g)$ is an automorphism of $\mathbb{P}^{n}$, we have $\alpha(g)^{*} H \sim H$. This implies that $g^{*} P \sim P$. Hence $g$ is of null entropy by Lemma 3.6.

For the 'only if' part: Suppose that $G$ is infinite and of null entropy. By Lemma 2.7, $G \mid L$ is also infinite. By Lemma 3.7, there exists a unique nonzero ray $\mathbb{R}_{>0}[D] \subseteq$ $\partial \mathcal{C}\left(L_{\mathbb{R}}\right) \cap \mathrm{NS}_{\mathbb{R}}(X)$ such that $g^{*} D \equiv D$ (i.e., $g^{*} D \sim D$ in the current case) for any $g \in G$. Further, $q_{X}(D)=0$ and $D$ can be chosen to be an integral divisor, where $q_{X}$ is the Beauville-Bogomolov quadratic form. Moreover, $D$ is in the closed movable cone $\overline{\operatorname{Mov}}(X)$ of $X$ and hence also in the closure of birational Kähler cone $\overline{\mathcal{B K}}(X)$ of $X$.

Now by [21, Corollary 1.1], $D$ gives rise to a rational Lagrangian fibration $\phi: X \rightarrow \mathbb{P}^{n}$, i.e., there exists a birational map $\tau: X \rightarrow X^{\prime}$ to another hyperkähler manifold $X^{\prime}$ such that the linear system $\left|D^{\prime}\right|$ gives rise to a Lagrangian fibration $\phi^{\prime}: X^{\prime} \rightarrow \mathbb{P}^{n}$ which is just the Stein factorization of

$$
\Phi_{\left|D^{\prime}\right|}: X^{\prime} \rightarrow \mathbb{P}\left(H^{0}\left(X^{\prime}, D^{\prime}\right)\right)
$$

where $D^{\prime}:=\tau_{*} D$ and $\phi=\phi^{\prime} \circ \tau$. Equivalently, we may also replace $D^{\prime}$ by a primitive class such that $\Phi_{\left|D^{\prime}\right|}$ itself is already a Lagrangian fibration, using the fact that $\operatorname{Pic} \mathbb{P}^{n} \cong \mathbb{Z}$. Replacing $(X, D)$ by $\left(X^{\prime}, D^{\prime}\right)$ and $G$ by $\tau G \tau^{-1}$, we may assume that $\Phi_{|D|}: X \rightarrow \mathbb{P}^{n}$ is already a holomorphic Lagrangian fibration. We only have to show that $G \leq \operatorname{Bir}(X)$ descends to a regular group action on $\mathbb{P}^{n}$. This is true because $g^{*} D \sim D$ for all $g \in G$ implies that $g$ induces an isomorphism of $\mathbb{P}^{n}=\mathbb{P}\left(H^{0}(X, D)\right)$. This proves Theorem 1.1 .

Part (1) below is contained in [5, Theorem 5.1], [6, Theorem 3.2] or [29, Theorem 3.1]. For commutative group actions in higher dimension; see [9, Theorem 1.1]. For an automorphism $g$ of a variety $X$, denote by $\operatorname{Per}(g)$ the set of $g$-periodic points, i.e., the set of points $x \in X$ such that $g^{s}(x)=x$ for some integer $s>0$ depending on $x$.

Theorem 4.3 (cf. [6, Theorem 3.2], [5, Theorem 5.1], or [29, Theorem 3.1]). Let X be a smooth projective surface. Assume $D$ is a numerically nonzero $\mathbb{R}$-divisor such that $D^{2} \geq 0$ (this holds when $D$ is nef). Assume further that $g_{i} \in \operatorname{Aut}(X)(i=1,2)$ are of positive entropy and polarized by $D$. Then we have:

(1) $g_{1}^{s_{1}}=g_{2}^{s_{2}}$ holds in $\operatorname{Aut}(X) \mid \mathrm{NS}(X)$ for some $s_{i} \in \mathbb{Z} \backslash\{0\}$.

(2) Suppose that either $\operatorname{Per}\left(g_{1}\right) \cap \operatorname{Per}\left(g_{2}\right) \neq \emptyset$, or $X$ is not birational to an abelian surface. Then $g_{1}^{t_{1}}=g_{2}^{t_{2}}$ holds in $\operatorname{Aut}(X)$ for some $t_{i} \in \mathbb{Z} \backslash\{0\}$. 
Proof. Let $L:=\mathrm{NS}(X) /$ (torsion) and $G=\left\langle g_{1}, g_{2}\right\rangle$. By assumption $[D] \in \overline{\mathcal{C}}\left(L_{\mathbb{R}}\right) \backslash\{0\}$ is a common eigenvector of $G \mid L$. So by Lemma 3.9, replacing $G$ by a finite-index subgroup, we have $N(G \mid L)$ is a subgroup of $G \mid L$ and $G / N(G) \cong(G \mid L) /(N(G \mid L)) \cong \mathbb{Z}$. Note that $N(G)$ is the inverse of $N(G \mid L)$ via the natural homomorphism $G \rightarrow G \mid L \leq \mathrm{O}(L)^{\prime}$, and $N(G) \mid L=N(G \mid L)$ is finite by Lemma 3.8. Replacing $g_{i}$ by powers, we may assume that $g_{1}=g_{2}$ (modulo $N(G)$ ). Hence part (1) follows from Lemma 2.4.

For part (2), since $G \neq N(G)$ and by [4, Proposition 1], we may assume that either $X$ is a rational surface, or $X$ is a minimal surface which is either a $K 3$ surface, an Enriques surface or an abelian surface.

Note that $N(G \mid L)$ is finite. Take an ample divisor $H^{\prime}$ on $X$ and set

$$
[H]:=\sum_{c \in N(G \mid L)} c^{*}\left[H^{\prime}\right]
$$

Then

$$
N(G) \leq \operatorname{Aut}_{[H]}(X):=\left\{g \in \operatorname{Aut}(X): g^{*}[H]=[H]\right\} .
$$

Since $\left|\operatorname{Aut}_{[H]}(X): \operatorname{Aut}_{0}(X)\right|<\infty$ by [12, Theorem 4.8] or [19, Proposition 2.2], we have $\left|N(G): N(G) \cap \operatorname{Aut}_{0}(X)\right|<\infty$. If $\operatorname{Aut}_{0}(X)=1$ (this is true when $X$ is a $K 3$ surface or an Enriques surface), then $N(G)$ is finite and hence part (2) follows from Lemma 2.4. We may assume that $\operatorname{Aut}_{0}(X) \neq 1$. This assumption and $G \neq N(G)$ imply that $X$ is not a rational surface (cf. Lemma 2.8).

Finally, we assume that $X$ is an abelian surface and $\operatorname{Per}\left(g_{1}\right) \cap \operatorname{Per}\left(g_{2}\right) \neq \emptyset$. Replacing $g_{i}$ by some common power, we may assume that both $g_{i}$ fix one common point $P \in X$. Note that for any $s \in \mathbb{N}$, we have $c_{s}:=g_{1}^{s} g_{2}^{-s} \in N(G) \leq \operatorname{Aut}_{[H]}(X)$. Since $\mid N(G)$ : $N(G) \cap \operatorname{Aut}_{0}(X) \mid<\infty$, we have $c_{s}=c_{t}$ (modulo $\left.N(G) \cap \operatorname{Aut}_{0}(X)\right)$ for some $t>s$. This implies that

$$
c_{s} c_{t}^{-1} \in N(G) \cap \operatorname{Aut}_{0}(X) \leq \operatorname{Aut}_{0}(X) .
$$

Now since $\operatorname{Aut}_{0}(X)$ consists of translations and $c_{s} c_{t}^{-1}$ fixes the point $P$, we have $c_{s} c_{t}^{-1}=\mathrm{id}$. So $g_{1}^{t-s}=g_{2}^{t-s}$ in $\operatorname{Aut}(X)$. This proves Theorem 4.3.

Proof of Theorem 1.2. Apply Proposition 3.13 to $L:=\mathrm{NS}(X)$ (also need Lemma 2.7 and Lemma 2.4).

Remark 4.4. For the converse direction of Theorem 1.2, assume that both $g_{i}$ in $\operatorname{Bir}(X)$ are of positive entropy such that $g_{1}^{t_{1}}=g_{2}^{t_{2}}$ for some $t_{i} \in \mathbb{Z} \backslash\{0\}$. Replacing $g_{i}$ by $g_{i}^{-1}$ if necessary, we may assume that both $t_{i}>0$.

Since $g_{1}$ preserves the closed movable cone $\overline{\operatorname{Mov}}(X)$ (see 2.1) of $X$ and this cone spans $\mathrm{NS}_{\mathbb{R}}(X)$, the generalized Perron-Frobenius theorem in [2] (see also Lemma 3.4) implies that $g_{1}^{*} D \equiv \lambda_{1} D$ for some movable $\mathbb{R}$-divisor $D$ and with $\log \lambda_{1}>0$ the entropy of $g_{1}$. Thus $\left(g_{2}^{t_{2}}\right)^{*} D \equiv \lambda D$ with $\lambda=\lambda_{1}^{t_{1}}>1$. Now the uniqueness result in Lemma 3.6 for $g_{2}^{t_{2}}$, implies that $g_{2}^{*} D \equiv \lambda_{2} D$ with $\log \lambda_{2}$ the entropy of $g_{2}$ and $\lambda_{2}^{t_{2}}=\lambda$.

If both $g_{i}$ are automorphisms, we can take $D$ to be a nef divisor.

The above argument also applies to surface automorphisms, so as to get the converse to Theorem $4.3(1)$. 
Proof of Proposition 1.3. Denote by $L:=\mathrm{NS}(X) /($ torsion) so that $G \mid L$ is infinite by the assumption. Hence the first assertion is just [23, Theorem 2.1]. Replacing $G$ by a finite-index subgroup, we may assume that $G \mid L \cong \mathbb{Z}^{\oplus s}$ for some $1 \leq s \leq \rho(X)-2$.

We still have to give a better upper bound for $s$. Note that the Kodaira dimension $\kappa(X) \leq 1$ since $G \mid L$ and hence $G$ are infinite. If $\kappa(X) \geq 0$, we descend $G$ to a biregular action on the minimal model of $X$ and may assume that $X$ is already minimal, i.e., the canonical divisor $K_{X}$ is nef. If $\kappa(X)=0$, by the classification theory of surfaces the Picard number $\rho(X) \leq h^{1,1}(X) \leq 20$ (with the equality possibly holds only when $X$ is a $K 3$ surface), so the proposition follows in this case.

If $\kappa(X)<0, X$ is either a rational surface or an irrational ruled surface.

If $X$ is an irrational ruled surface, replacing $G$ by a finite-index subgroup, we may assume that $G$ descends to a biregular action on a relatively minimal model $X_{m}$, after $G$-equivariant blowdowns of -1 -curves in the unique $\mathbb{P}^{1}$-fibration on $X$; replacing $X$ by $X_{m}$, we may assume that $X$ is already relatively minimal. Now this $X$ has Picard number two, both extremal rays of the nef cone of $X$ are fixed by $G$, contradicting the uniqueness of the $G$-stable nef ray in Lemma 3.7.

If $X$ is rational, we may assume that the pair $(X, G)$ is minimal and hence $K_{X}^{2}=0$ by [13, Theorem 2]. Thus the Picard number of $X$ is 10 and hence $s \leq 8$. This proves the proposition.

\section{REFERENCES}

[1] A. Bayer and E. Macrì, MMP for moduli of sheaves on K3s via wall-crossing: nef and movable cones, Lagrangian fibrations, Invent. Math. 198 (2014), no. 3, 505-590.

[2] G. Birkhoff, Linear transformations with invariant cones, Amer. Math. Monthly 74 (1967), 274-276.

[3] F. Campana, F. Wang, and D. -Q. Zhang, Automorphism groups of positive entropy on projective threefolds, Trans. Amer. Math. Soc. 366 (2014), no. 3, 1621-1638.

[4] S. Cantat, Dynamique des automorphismes des surfaces projectives complexes, C. R. Acad. Sci. Paris Sér. I Math. 328 (1999), no. 10, 901-906.

[5] _ Sur les groupes de transformations birationnelles des surfaces, Ann. of Math. (2) 174 (2011), no. $1,299-340$.

[6] — Dynamics of automorphisms of compact complex surfaces, in "Frontiers in Complex Dynamics: In celebration of John Milnor's 80th birthday", volume 51 of Princeton Math. Series, pp. 463-514, Princeton University Press, 2014.

[7] J. Diller and C. Favre, Dynamics of bimeromorphic maps of surfaces, Amer. J. Math. 123 (2001), no. $6,1135-1169$.

[8] T. -C. Dinh, Tits alternative for automorphism groups of compact Kähler manifolds, Acta Math. Vietnam 37 (2012), no. 4, 513-529.

[9] T. -C. Dinh and N. Sibony, Groupes commutatifs d'automorphismes d'une variété kählérienne compacte, Duke Math. J. 123 (2004), no. 2, 311-328.

[10] C. Favre, Le groupe de Cremona et ses sous-groupes de type fini, Séminaire Bourbaki. Volume 2008/2009. Exposés 997-1011. Astérisque No. 332 (2010), Exp. No. 998, vii, 11-43.

[11] B. Fu and D. -Q. Zhang, A characterization of compact complex tori via automorphism groups, Math. Ann. 357 (2013), no. 3, 961-968.

[12] A. Fujiki, On automorphism groups of compact Kähler manifolds, Invent. Math. 44 (1978), no. 3, $225-258$. 
[13] M. Kh. Gizatullin, Rational G-surfaces, Math. USSR Izv. 16 (1981), no. 1, 103-134.

[14] M. Gromov, On the entropy of holomorphic maps, Enseign. Math. (2) 49 (2003), no. 3-4, 217-235.

[15] D. Guan, On the Betti numbers of irreducible compact hyperkähler manifolds of complex dimension four, Math. Res. Lett. 8 (2001), no. 5-6, 663-669.

[16] D. Huybrechts, Compact hyperkähler manifolds: basic results, Invent. Math. 135 (1999), no. 1, 63-113.

[17] _ Compact hyperkähler manifolds, Calabi-Yau manifolds and related geometries (Nordfjordeid, 2001), Universitext, Springer, Berlin, 2003, pp. 161-225.

[18] J. Keum, K. Oguiso, and D. -Q. Zhang, Conjecture of Tits type for complex varieties and theorem of Lie-Kolchin type for a cone, Math. Res. Lett. 16 (2009), no. 1, 133-148.

[19] D. I. Lieberman, Compactness of the Chow scheme: applications to automorphisms and deformations of Kähler manifolds, Fonctions de plusieurs variables complexes, III (Sém. François Norguet, 19751977), Lecture Notes in Math., vol. 670, Springer, Berlin, 1978, pp. 140-186.

[20] E. Markman, Lagrangian fibrations of holomorphic-symplectic varieties of $K 3^{[n]}$-type, Proceedings of the Conference Algebraic and Complex Geometry held at Hannover, September 10-14, 2012, In Honour of Klaus Hulek's 60th Birthday, Springer Proc. Math. Stat., vol. 71, Springer, Cham, 2014, pp. 241-283.

[21] D. Matsushita, On isotropic divisors on irreducible symplectic manifolds, (2013), arXiv:1310.0896.

[22] K. Oguiso, Tits alternative in hypekähler manifolds, Math. Res. Lett. 13 (2006), no. 2-3, 307-316.

[23] _ Automorphisms of hyperkähler manifolds in the view of topological entropy, Algebraic geometry, Contemp. Math., vol. 422, Amer. Math. Soc., Providence, RI, 2007, pp. 173-185.

[24] _ Bimeromorphic automorphism groups of non-projective hyperkähler manifolds - a note inspired by C. T. McMullen, J. Differential Geom. 78 (2008), no. 1, 163-191.

[25] _ Simple abelian varieties and primitive automorphisms of null entropy of surfaces, (2014), arXiv: 1412.2535.

[26] J. Ratcliffe, Foundations of hyperbolic manifolds, second ed., Graduate Texts in Mathematics, vol. 149, Springer, New York, 2006.

[27] Y. Yomdin, Volume growth and entropy, Israel J. Math. 57 (1987), no. 3, 285-300.

[28] K. Yoshioka, Bridgeland's stability and the positive cone of the moduli spaces of stable objects on an abelian surface, (2012), arXiv:1206.4838.

[29] D. -Q. Zhang, Automorphism groups and anti-pluricanonical curves, Math. Res. Lett. 15 (2008), no. $1,163-183$.

[30] _ Dynamics of automorphisms on projective complex manifolds, J. Differential Geom. 82 (2009), no. 3, 691-722.

[31] _ A theorem of Tits type for compact Kähler manifolds, Invent. Math. 176 (2009), no. 3, $449-459$.

Department of Mathematics

National University of Singapore, 10 Lower Kent Ridge Road, Singapore 119076

E-mail address: hf@u.nus.edu

School of Mathematics

Korea Institute for Advanced Study, Seoul 130-722, Korea

E-mail address: jhkeum@kias.re.kr

Department of Mathematics

National University of Singapore, 10 Lower Kent Ridge Road, Singapore 119076

E-mail address: matzdq@nus.edu.sg 